\title{
Evaluation of leaf proteomics responses during selenium mediated tolerance of arsenic toxicity in rice (Oryza sativa L.)
}

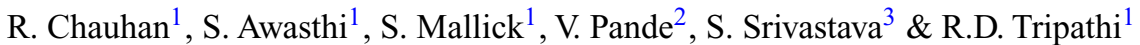 \\ ${ }^{1}$ CSIR - National Botanical Research Institute, Lucknow, Uttar Pradesh, India \\ ${ }^{2}$ Department of Biotechnology, Kumaun University, Bhimtal, Nainital, Uttarakhand, India \\ ${ }^{3}$ Institute of Environment \& Sustainable Development, Banaras Hindu University, Varanasi, Uttar Pradesh, India
}

\begin{abstract}
Arsenic (As) is a toxic element, which poses significant threat to human health due to frequency of occurrence, toxicity and various routes for human exposure. Arsenic contamination of water and food, especially rice, is a serious issue. Selenium (Se) is an essential element for human and other animals and is beneficial for plants as well. Selenium has been found to antagonize the toxicity of As in plants. The proteomics study was performed by using 2-dimensional gel electrophoresis and MALDI-TOF/TOF. The present study provides new insights into As-Se interaction in rice leaves at the proteome level and identifies crucial proteins involved in Se mediated amelioration of As toxicity in rice.
\end{abstract}

\section{INTRODUCTION}

Arsenic (As) is an environmental toxin and class I human carcinogen. Arsenic contamination is a major environmental hazard of recent times which poses a health risk for human through food chain contamination. Nearly 200 million people are at risk of As toxicity. Rice is the dietary staple for half of the world's population and a good accumulator of As. Thus, rice consumers are at great risk. Arsenic exerts toxicity to plants in various ways and hampers plants growth. Increased production of reactive oxygen species (ROS) and oxidative damage to plants are apparent symptoms of As toxicity. Selenium (Se) is an essential nutrient element for human and is also beneficial element for plants which enables them to counter against various abiotic stresses at low dosages. Selenium acts as an antioxidant and is involved in the detoxification of heavy metals including As by alleviating the oxidative stress and antagonizing the uptake of heavy metals (Chauhan et al., 2017). Thus, supplementation of Se in As contaminated environment might be an important strategy to reduce As uptake and associated phytotoxicity in rice plant. The present study analyzes As-Se interaction in rice leaves at the proteome level.

\section{METHODS}

\subsection{Plant material and experimental conditions}

Rice variety, Triguna was used for the experiments. Rice seedlings were exposed to As and Se for 15 days using sodium arsenite ( $\mathrm{As}(\mathrm{III}), \mathrm{NaAsO}_{2}$ ) and sodium selenite ( $\mathrm{Se}(\mathrm{IV}), \mathrm{Na}_{2} \mathrm{SeO}_{3}$ ). Plants were exposed to different treatments; control, $25 \mu \mathrm{M}$ As(III), $25 \mu \mathrm{M}$ $\mathrm{Se}(\mathrm{IV})$ and $25 \mu \mathrm{M}(\mathrm{As}+\mathrm{Se})$ for 15 days. After $15 \mathrm{~d}$ exposure, the plants were harvested in triplicate for proteome analysis and As accumulation.

\subsection{Protein extraction, 2DE analysis, image analysis, protein identification and MALDI-TOF-TOF analysis}

Rice leaf proteins from three biological replicates were extracted using ammonium acetate/acetone precipitation method followed by phenol extraction as per method described by Agrawal et al. (2008). Total shoot protein was determined by Bradford assay (Bio-Rad, USA). The $13 \mathrm{~cm}$ IEF strips ( $\mathrm{pH} 4-7$ ) were rehydrated with protein $(250 \mu \mathrm{g})$ for $16 \mathrm{~h}$ and electrofocused using an IPGphor system (Bio-Rad, USA) at $20^{\circ} \mathrm{C}$ up to $25,000 \mathrm{Vh}$. After reduction with DTT and alkylation by using IAA, the strips were then loaded on top of $12.5 \%$ poly acrylamide gels for SDS-PAGE. The gels were fixed and stained with silver stain (silver stain plus kit; Bio-Rad, USA). The silver stained gel obtained after 2DE were scanned for image acquisition using the Bio-Rad FluorS system equipped with a 12-bit camera. Images from three biological replicate 2-DE gels were taken for the data analysis in PD Quest version 8.0.1 (Bio-Rad). Protein spots showing significant changes in abundance between different treatments were selected and excised manually and processed for peptide identification by MALDI-TOFTOF (Model 4800, Applied Biosystems, USA). In all the protein identifications, probability scores were greater than the score fixed by Mascot as significant with a $\mathrm{p}<0.05$.

\subsection{Arsenic and selenium quantification}

For estimation of total As and Se, dried plant samples $(0.2 \mathrm{~g})$ were powdered and digested in $3 \mathrm{~mL} \mathrm{HNO}_{3}$ at $120^{\circ} \mathrm{C}$ for $6 \mathrm{~h}$ (Dwivedi et al., 2010). Total As and Se 
were quantified with the help of inductively coupled plasma mass spectrometer (ICP-MS, Agilent $7500 \mathrm{cx}$ ). The multielement standard solution (Agilent, Part \# 8500-6940) was used for the calibration and quality assurance for each analytical batch. Known concentrations of spiked samples were prepared to check the As and Se recovery. Selenium and As were recovered about $93.5 \%( \pm 2.3 ; \mathrm{n}=5)$ and $92.5 \%( \pm 3.1 ; \mathrm{n}=5)$, respectively.

\section{RESULTS AND DISCUSSION}

\subsection{Expression profile of rice leaf proteins}

In the present study, the protein expression profile of rice leaf proteins under As stress and Se supplementation has been investigated that provides a fine picture of protein networks and metabolic pathways involved in cellular detoxification and tolerance mechanism. Results showed that Se supplementation (25 $\mu \mathrm{M} \mathrm{Se}(\mathrm{IV}))$ to As $(25 \mu \mathrm{M} \mathrm{As}(\mathrm{III}))$ exposed rice plants improved the plant growth in comparison to As alone treatment, exhibiting antagonistic interaction between As and Se. Photosynthetic pigments were also significantly increased upon Se supplementation during As stress. The improved growth of rice in $\mathrm{As}+\mathrm{Se}$ treatment was thus attributable to the improved levels of photosynthetic pigments which contribute towards biomass gain and in addition to lesser As uptake. By comparison of gel images of different treatments viz., control, As and Se with the help of PDQuest software, 145 differentially regulated spots were subjected for MALDI-TOF MS-MS analysis out of which 110 protein spots identified which further led to identification of 85 proteins with a significant score (Fig. 1). Functional categorization and cluster analysis (SOTA analysis) of As and Se responsive differentially expressed proteins revealed proteins of defense, photosynthesis, ROS pathway, energy metabolism, antioxidant system, amino acid metabolism and transport to be the responsive proteins. Protein ubiquitination and phosphorylation also appeared to play important roles in Se mediated amelioration of As toxicity in rice. In conclusion, the present study provides new insights into As-Se interaction in rice plant at the proteome level and identifies crucial proteins involved in Se mediated As toxicity amelioration in rice.

\subsection{Effect of selenium on arsenic accumulation}

When Se and As were supplied together the accumulation of As was reduced significantly $(p<0.05)$ in both roots and shoots. The maximum reduction in As accumulation was also observed at equimolar concentration of $\mathrm{As}(\mathrm{III})$ and $\mathrm{Se}(\mathrm{IV})(25 \mu \mathrm{M})$ with about $68 \%$ and $45 \%$ reduction in roots and shoots respectively in comparison to As(III) alone treated plants. On the contrary, the accumulation of Se increased in both the tissues of rice plant. Thus, Se helps in reducing As uptake and subsequently increase uptake of other divalent cations required for antioxidant defense mechanism.

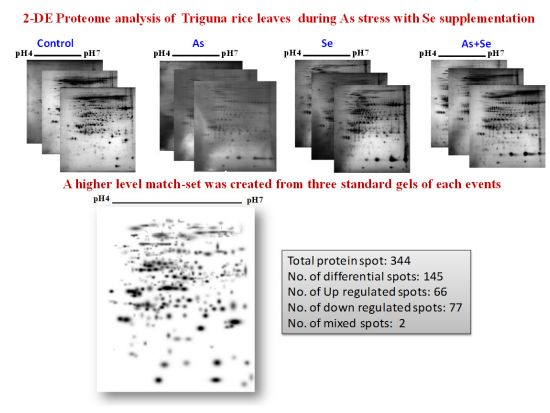

Figure 1. Representation of 2DE maps of rice leaf proteins isolated from control, As, Se and As + Se treatments. Three replicate silver stained gels for each stage were computationally combined using PDQuest software and one representative master standard gel images were generated.

\section{CONCLUSIONS}

Present study with the help of comparative proteomic study provides a systemic molecular overview of Se mediated modulation of As toxicity in rice plant. Functional classification revealed that maximum number of proteins fall in the category of bioenergy and metabolism followed by those involved in cell defense. Photosynthesis is the most affected biological process of rice shoot by As treatment. Selenium supplementation repaired the photosynthetic system and the proteins involved in photosynthesis were found upregulated in Se treatment during As stress. Stress responses and cell redox homeostasis were also found enhanced on Se supplementation. Selenium treatment also activated antioxidant system to enhance As resistance in rice plant. Present proteomics study could represent only a small part of the rice proteome during As and Se interaction, further investigation to assign their putative biological functions may be useful for a better understanding of complex biological traits.

\section{ACKNOWLEDGEMENT}

Reshu Chauhan is thankful to Department of Science and Technology (DST) New Delhi, India for the award of DST-INSPIRE Junior/Senior Research Fellowship.

\section{REFERENCES}

Agrawal, L., Chakraborty, S., Jaiswal, D.K., Gupta, S., Datta, A. \& Chakraborty, N. 2008. Comparative proteomics of tuber induction, development and maturation reveal the complexity of tuberization process in potato (Solanum tuberosum L.). J. Proteome Res. 7(9): 3803-3817.

Chauhan, R., Awasthi, S., Tripathi, P., Mishra, S., Dwivedi, S., Niranjan, A., Mallick, S., Tripathi, P., Pande, V. \& Tripathi, R.D. 2017. Selenite modulates the level of phenolics and nutrient element to alleviate the toxicity of arsenite in rice (Oryza sativa L.). Ecotox. Environ. Saf. 138: 47-55.

Dwivedi, S., Tripathi, R., Tripathi, P., Kumar, A., Dave, R., Mishra, S., Singh, R., Sharma, D., Rai, U. \& Chakrabarty, D. 2010. Arsenate exposure affects amino acids, mineral nutrient status and antioxidant in rice (Oryza sativa L.) genotypes. Environ. Sci. Technol. 44: 9542-9549. 